\title{
Picosecond dynamics of internal exciton transitions in CdSe nanorods
}

Cooke, D. G.; Jepsen, Peter Uhd; Lek, Jun Yan; Lam, Yeng Ming; Sy, F.; Dignam, M. M.

Published in:

Physical Review B Condensed Matter

Link to article, DOI:

10.1103/PhysRevB.88.241307

Publication date:

2013

Document Version

Publisher's PDF, also known as Version of record

Link back to DTU Orbit

Citation (APA):

Cooke, D. G., Jepsen, P. U., Lek, J. Y., Lam, Y. M., Sy, F., \& Dignam, M. M. (2013). Picosecond dynamics of internal exciton transitions in CdSe nanorods. Physical Review B Condensed Matter, 88(24), [241307]. https://doi.org/10.1103/PhysRevB.88.241307

\section{General rights}

Copyright and moral rights for the publications made accessible in the public portal are retained by the authors and/or other copyright owners and it is a condition of accessing publications that users recognise and abide by the legal requirements associated with these rights.

- Users may download and print one copy of any publication from the public portal for the purpose of private study or research.

- You may not further distribute the material or use it for any profit-making activity or commercial gain

- You may freely distribute the URL identifying the publication in the public portal 


\title{
Picosecond dynamics of internal exciton transitions in CdSe nanorods
}

\author{
D. G. Cooke* \\ Department of Physics, McGill University, Montreal, Quebec, Canada H3A2T8 \\ P. Uhd Jepsen \\ Department of Photonics Engineering, Technical University of Denmark, 2800 Kgs. Lyngby, Denmark \\ Jun Yan Lek \\ School of Materials Science and Engineering, Nanyang Technological University, 639798, Singapore \\ Yeng Ming Lam \\ School of Materials Science and Engineering, Nanyang Technological University, 639798, Singapore \\ and Institute of Materials for Electronic Engineering II, RWTH-Aachen, Sommerfeldstr. 24, D-52074 Aachen, Germany \\ F. Sy and M. M. Dignam \\ Department of Physics, Engineering Physics and Astronomy, Queen's University, Kingston, Ontario, Canada K7L 3N6 \\ (Received 6 August 2013; published 30 December 2013)

\begin{abstract}
The picosecond dynamics of excitons in colloidal CdSe nanorods are directly measured via their $1 s$ to $2 p$-like internal transitions by ultrabroadband terahertz spectroscopy. Broadened absorption peaks from both the longitudinal and transverse states are observed at 8.5 and $11 \mathrm{THz}$, respectively. The onset of exciton-LO phonon coupling appears as a bleach in the optical conductivity spectra at the LO phonon energy for times $>1 \mathrm{ps}$ after excitation. Simulations show a suppressed exciton temperature due to thermally excited hole states being rapidly captured onto ligands or unpassivated surface states. The relaxation kinetics are manipulated and the longitudinal transition is quenched by surface ligand exchange with hole capturing pyridine.
\end{abstract}

DOI: 10.1103/PhysRevB.88.241307

PACS number(s): 78.67.Qa, 63.20.kk, 71.35.Cc, 78.47.jh

Quantum dots are one of the essential building blocks of nanoscience and hold great promise for future optoelectronic applications. ${ }^{1}$ Their optical properties are conveniently tailored by quantum confinement of the exciton. ${ }^{2}$ The dynamics of exciton relaxation in colloidal quantum dots often defines their limitations for a variety of solution-processed optoelectronic devices, ranging from lasers to spin-cast solar cells and so is currently a topic of intense research. ${ }^{3}$ Our understanding of these processes relies largely on the interrogation of interband transitions in the optical regime, which can be understood in the independent electron and hole picture provided multiexciton effects are negligible and the nanoparticles are charge neutral prior to illumination. This approximation is valid for particle sizes less than the exciton Bohr radius where the particle confinement energies well exceed the Coulomb attraction of the electron and hole. For larger or strongly asymmetric particles, such as nanorods, this approach is no longer valid, and the interpretation of these spectra becomes more difficult.

Time-resolved $\mathrm{THz}$ spectroscopy has made it possible to directly probe excitons in bulk semiconductors and 2D quantum wells through their internal transitions on a $1-10 \mathrm{meV}$ scale. ${ }^{4-6}$ Excitons in colloidal quantum dot and rod systems, however, are expected to have internal transitions in the $10-50 \mathrm{meV}$ range. $\mathrm{THz}$ spectroscopy in the $0.8-12 \mathrm{meV}$ band has been previously used to probe the off-resonant polarizability, ${ }^{7,8}$ long range coupling, ${ }^{9}$ and energy transfer between electrons and holes in quantum dots. ${ }^{10}$ Midinfrared femtosecond spectroscopy has been applied to probe internal electronic transitions in strongly confined CdSe quantum dots where the Coulomb interaction with the hole is a small perturbation and the electronic transitions are well defined on a few $100 \mathrm{meV}$ scale. ${ }^{11}$ These photon energies, however, are too large to probe the ground state transitions of the exciton in larger particles such as nanorods.

In this Rapid Communication, we apply time-resolved multi- $\mathrm{THz}$ spectroscopy in the $1-13 \mathrm{THz}(1-50 \mathrm{meV})$ range to probe exciton relaxation dynamics in $\mathrm{CdSe}$ colloidal nanorods capped with organic ligands by probing their internal excitonic transitions. We observe intraexcitonic transitions in nanorods and we see evidence of rapid phonon-induced thermalization in a subset of the rod population. Two competing channels for exciton relaxation are observed: capture to surface states on a 4 ps time scale which can be manipulated through ligand exchange and recombination occurring on a longer time scale of several tens of ps. This work demonstrates that multi-THz spectroscopy is a powerful tool to investigate exciton dynamics in colloidal nanoparticles and paves the way for future studies of state-dependent relaxation pathways, ${ }^{12}$ electron-phonon coupling, ${ }^{13}$ and even manipulation of excitons in nanoparticles by strong $\mathrm{THz}$ fields. ${ }^{14}$

The samples studied are CdSe nanorods $7 \pm 1 \mathrm{~nm}$ in diameter and $70 \pm 10 \mathrm{~nm}$ in length, synthesized using the hot coordinating solvents method described elsewhere. ${ }^{15}$ The nanorods exhibit an absorption edge at approximately $685 \mathrm{~nm}$ and a weak photoluminesence peak at $683 \mathrm{~nm}$ with a FWHM of $30 \mathrm{~nm}$. The surfaces of the nanorods are passivated with the organic ligand dodecylphosphonic acid (DDPA), which at $\sim 2 \mathrm{~nm}$ in length serves to electrically isolate the nanorods. ${ }^{9}$ These particles were subsequently drop cast from solution 
onto indium tin oxide (ITO) coated glass slides with a sheet resistivity of $2 \Omega / \mathrm{sq}$, which reflects more than $98 \%$ of the $\mathrm{THz}$ field amplitude over the entire $\mathrm{THz}$ pulse bandwidth. The thickness of the film varied between 1 and $1.6 \mu \mathrm{m}$.

The experimental setup is described in more detail elsewhere, ${ }^{16}$ but briefly, we employ an ultrabroadband timeresolved $\mathrm{THz}$ spectrometer operating in reflection mode based on two-color laser plasma $\mathrm{THz}$ generation ${ }^{17}$ and air-biased coherent detection (ABCD). ${ }^{18}$ The spectrometer is driven by $35 \mathrm{fs}, 800 \mathrm{~nm}$ pulses provided at $1 \mathrm{kHz}$ repetition rate by a Ti:sapphire regenerative amplifier. The sample was placed at normal incidence in the focus of the $\mathrm{THz}$ pulse and the reflection was directed via a high resistivity $\mathrm{Si}$ beamsplitter to the ABCD module for detection. A double modulation scheme is used with two lock-in amplifiers to simultaneously detect the $\mathrm{THz}$ transient reflected in the presence of the pump $E_{\text {pump }}(t, \Delta t)$ and the differential transient $\Delta E(t, \Delta t)=$ $E_{\text {pump }}(t, \Delta t)-E_{\text {ref }}(t)$, where $E_{\text {ref }}(t)$ is the reflected $\mathrm{THz}$ transient in the absence of the photoexcitation and $\Delta t$ is the pump-probe delay time. ${ }^{19}$ The nanorods are photoexcited colinearly through a small hole in the last parabolic mirror before the sample by $400 \mathrm{~nm}$ fs laser pulses at a fluence of $570 \mu \mathrm{J} / \mathrm{cm}^{2}$, unless otherwise stated. All measurements are performed at room temperature in a dry nitrogen environment.

The differential reflectance can be processed into a frequency dependent optical density $\triangle \mathrm{OD}(\omega)=$ $-\ln \left[\left|E_{\text {pump }}(\omega)\right| /\left|E_{\text {ref }}(\omega)\right|\right]$, with the THz pulse traversing the thin film sample twice due to the reflection off the underlying ITO which enhances sensitivity. ${ }^{20}$ Figure 1 (a) shows $\triangle \mathrm{OD}(\omega)$ at 1 ps and 9 ps following photoexcitation. A broad absorption peak is observed at $8.5 \mathrm{THz}$ with a higher energy shoulder at approximately $11 \mathrm{THz}$. Also shown is the calculated intra-excitonic optical density spectrum (see below) scaled to fit the 1 ps data; the agreement is very good.

In all of the experimental spectra there is a small but noticeable reduction in the $\Delta \mathrm{OD}(\omega)$ spectra at approximately 6.2 $\mathrm{THz}$ that only appears for pump probe times greater than 1 ps [see Fig. 1(a)] and that does not appear in the theoretical excitonic absorption results of Fig. 2(a). In Fig. 1(b) we show the normalized difference between $\Delta t=9 \mathrm{ps}$ and $\Delta t=1$ ps absorbance spectra shown in Fig. 1(a), as well as the differential between the respective phase functions $\Delta \Phi(\omega)=$ $\arg \left[E_{\text {pump }}(\omega)\right]-\arg \left[E_{\text {ref }}(\omega)\right]$. A clear bleach peak is observed in the absorbance, accompanied by a phase flip at the CdSe longitudinal optical (LO) phonon energy at $205 \mathrm{~cm}^{-1}(6.15$ $\mathrm{THz}$ ). This bleach signal can be explained if we assume that some of the nanorods have an intraexcitonic transition energy (perhaps $1 s-2 p_{z}$ ) that is resonant with the LO phonon. In these nanorods, there will be a resonant Fröhlich interaction scattering excitons between the two transition states via the absorption of an LO phonon. ${ }^{13,21,22}$ This process will lead to a rapid thermal population of the upper state, which will result in the observed bleaching of the absorption at $6.2 \mathrm{THz}$. The linewidth of the bleach signal is $\sim 0.5 \mathrm{THz}$, consistent with previous measurements of the Raman linewidth in CdSe nanorods. ${ }^{23}$ The onset of exciton-phonon coupling has been previously observed and is attributed to rapid trapping of charge on the surface of the nanorod on a 1-10 ps time scale, causing an internal field which polarizes the exciton and leads to an increase in exciton-phonon coupling. ${ }^{13,22}$ For nanorods,

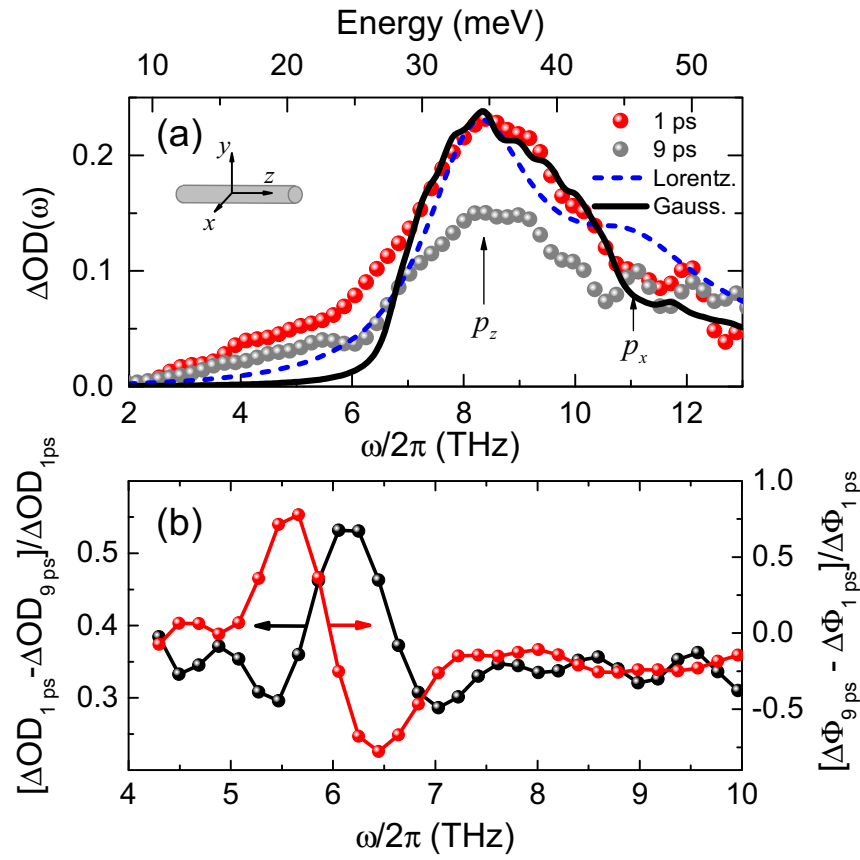

FIG. 1. (Color online) (a) Pump induced change in optical density, $\Delta \mathrm{OD}(\omega)$, of the CdSe nanorod film following $400 \mathrm{~nm}$ fs excitation at a pump-probe delay of $\Delta t=1 \mathrm{ps}$ and $9 \mathrm{ps}$. Also shown are calculated absorbance spectra for nanorods with a radius of $3.5 \mathrm{~nm}$ assuming a Lorentzian linewidth (blue dashed) and for a Gaussian distribution of nanorod radii (black solid) as described in the text. (b) The relative differential absorbance and phase between 9 ps and 1 ps pump-probe time delays, showing a bleaching signal at $6.2 \mathrm{THz}$.

however, such an internal field is not necessary to initiate the exciton-phonon coupling due to the asymmetric wave functions. Thus we attribute the onset to the thermalization of the excitons due to their $\sim 1.3 \mathrm{eV}$ excess energy compared to the ground state.

To calculate the CdSe nanorod absorbance spectrum arising from transitions between excitonic states, we first calculate the light and heavy hole excitonic energies and envelope functions by expanding them in a basis of noninteracting electron and hole nanorod states. We neglect the coupling between light holes ( $\mathrm{LH})$ and heavy holes $(\mathrm{HH})$ and calculate the electron-hole Coulomb interaction matrix elements to obtain the ground $1 s$ excitonic state as well as all of the excited states up to energies beyond the frequency response of the $\mathrm{THz}$ detector. In contrast to small quantum dots, the electron and hole states are greatly modified by the Coulomb interaction between them and we require a basis of 10 electron, $60 \mathrm{LH}$, and $60 \mathrm{HH}$ states to obtain convergence for the relevant excitonic states. We employ infinite confining potentials at the surfaces of the cylindrical nanorods and use effective masses given by the diagonal terms in the Luttinger matrix of Ref. 24. We take the nanorods to be cylindrical with a length of $70 \mathrm{~nm}$ and assume that there is at most one exciton per nanorod. It is assumed the nanorods all have their cylindrical axes parallel to the substrate surface; for a given nanorod, the direction parallel to the cylindrical axis is the $z$ direction and the radial direction in the plane of the substrate is the $x$ direction [see inset to Fig. 1(a)]. Because the LHs are heavier in the radial 

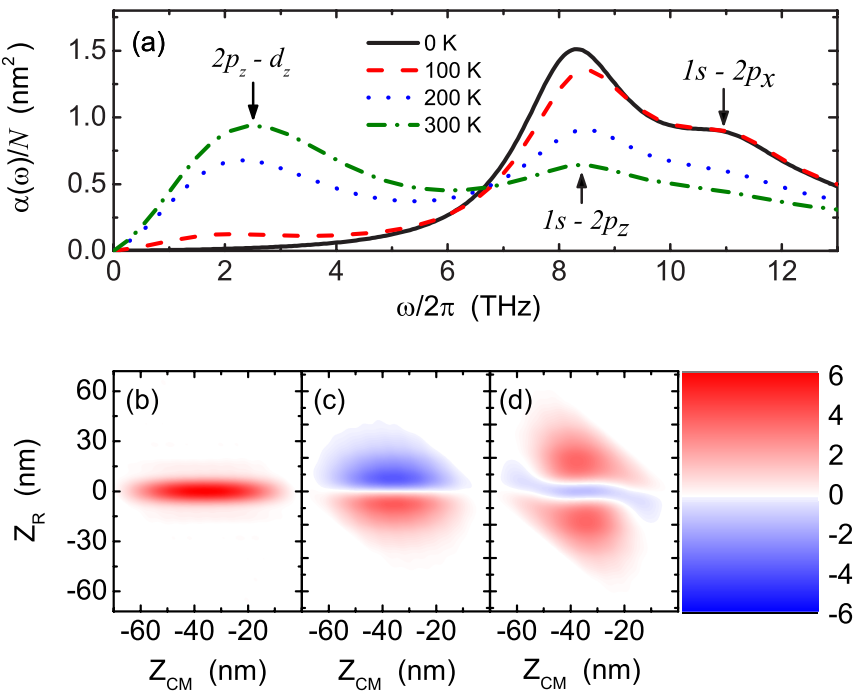

FIG. 2. (Color online) (a) Calculated absorbance spectra for four different temperatures for nanorods with a radius of $3.5 \mathrm{~nm}$, assuming a Lorentzian linewidth of $10 \mathrm{meV}$. The transitions for the three main peaks are indicated. The exciton wave function is plotted as a function of the center of mass $\left(Z_{\mathrm{CM}}\right)$ and relative $\left(Z_{R}\right)$ coordinate for the (b) $1 s$, (c) $2 p_{z}$, and (d) $d_{z}$ excitonic states.

direction, the lowest-energy exciton is the $1 s$ LH exciton, with the ground state $\mathrm{HH}$ exciton being $16.1 \mathrm{meV}$ higher in energy.

The dashed blue curve in Fig. 1(a) is produced assuming a rod radius of $3.5 \mathrm{~nm}$ and a Lorentzian linewidth of $2.4 \mathrm{THz}$ (10 meV) for all transitions and scaled to fit the main peak for the 1 ps data. To produce this spectrum, we assume that all excitons are in the $1 s \mathrm{LH}$ ground state when the $\mathrm{THz}$ pulse arrives. The main absorption peak at $8.3 \mathrm{THz}$ is due to the transition from the $1 s \mathrm{LH}$ exciton ground state to a $2 p_{z}$-like excitonic state. The $z$ dependence of these two states on the rod axis is shown in Figs. 2(b) and 2(c), respectively. The dipole matrix element for this transition is $D_{z} / e=3.0 \mathrm{~nm}$, where $e$ is the magnitude of the electron charge. The weaker transition, which appears as a shoulder at $10.8 \mathrm{THz}$ is due to a transition from the LH $1 s$ ground state to the LH $2 p_{x}$ state, which has a dipole moment of only $D_{x} / e=1.15 \mathrm{~nm}$. We note that there are many other excitonic states with excited center-of-mass motion with lower energies than the $2 p_{z}$ and $2 p_{x}$ states, but the transition dipole between these states and the ground state is negligible.

If we now assume that the excitons are in quasithermal equilibrium, we obtain the absorbance spectra shown in Fig. 2(a) for four different temperatures. As the temperature increases, the relative strengths of the $1 s-2 p_{z}$ and $1 s-2 p_{x}$ transitions change. In addition, above about $T=100 \mathrm{~K}$, a new peak appears at about $2.4 \mathrm{THz}$, which is largely due to a transition from the $\mathrm{LH} 2 p_{z}$ state to a higher $\mathrm{LH} d_{z}$-like state shown in Fig. 2(d); the dipole matrix element for this transition is $D_{p d} / e=12.0 \mathrm{~nm}$, which is why the peak is so large.

Reasonable agreement with experiment is only found if we assume an effective temperature of less than $100 \mathrm{~K}$. We interpret this result as a strong indication that only the ground state exciton has a significant population when the $\mathrm{THz}$ pulse hits the sample. We propose that the absence of excitons in excited states is due to the rapid transfer of the holes in excited excitonic states into traps in the ligand. As such trapped holes are spatially separate from the electrons in the nanorods, these "bound excitons"have negligible intraexcitonic dipole matrix elements and so do not contribute to the $\mathrm{THz}$ absorption.

Scanning electron microscopy (SEM) of the nanorod samples shows a distribution of nanorod diameters and length. For the range of lengths observed, the $\mathrm{THz}$ absorption shows little length dependence but significant dependence on radius. The solid black curve in Fig. 1(a) is produced taking the nanorod radii to have a Gaussian distribution, with a mean of $3.25 \mathrm{~nm}$ and standard deviation of $0.7 \mathrm{~nm}$, consistent with the values observed in SEM. To make the calculations tractable, we have discretized the distribution in steps of $0.05 \mathrm{~nm}$, which results in small oscillations seen in the curve. We have measured the thickness of the nanorod film in the measured spot to be $\sim 1.5 \mu \mathrm{m}$. The remaining unknown is the exciton density, treated as a fitting parameter and corresponding to 0.45 excitons per nanorod for the $\Delta t=1 \mathrm{ps}$ spectra. The agreement is quite good, except for frequencies below $\sim 6 \mathrm{THz}$, where a low frequency absorption tail is present. This may be due to contributions from excited state transitions or a non-Gaussian, large-radius tail in the actual nanorod radius distribution.

To investigate the influence of surface passivation, we have performed a ligand exchange with a hole scavenging ligand following the work of Guyot-Sionnest et al., where $70 \%$ of the DDPA is replaced by pyridine. ${ }^{11}$ Figure 3 shows there is a strong suppression of the excitonic $\triangle \mathrm{OD}$ peak and a slight blueshift to $9.8 \mathrm{THz}$. At this point there are no models for the influence of bound surface charge on the internal excitonic transitions of a nanorod. It appears that these states have lower energy than the excited excitonic states of the nanorods, as we see from comparison of the room temperature plot in Fig. 2(a) to the experimental results that there is essentially no evidence of populations in the excited excitonic states at room temperature. Also shown in Fig. 3 is the $\Delta \mathrm{OD}(\omega)$ spectra of the DDPA passivated nanorods at $\Delta t=1.0 \mathrm{ps}$ for a factor

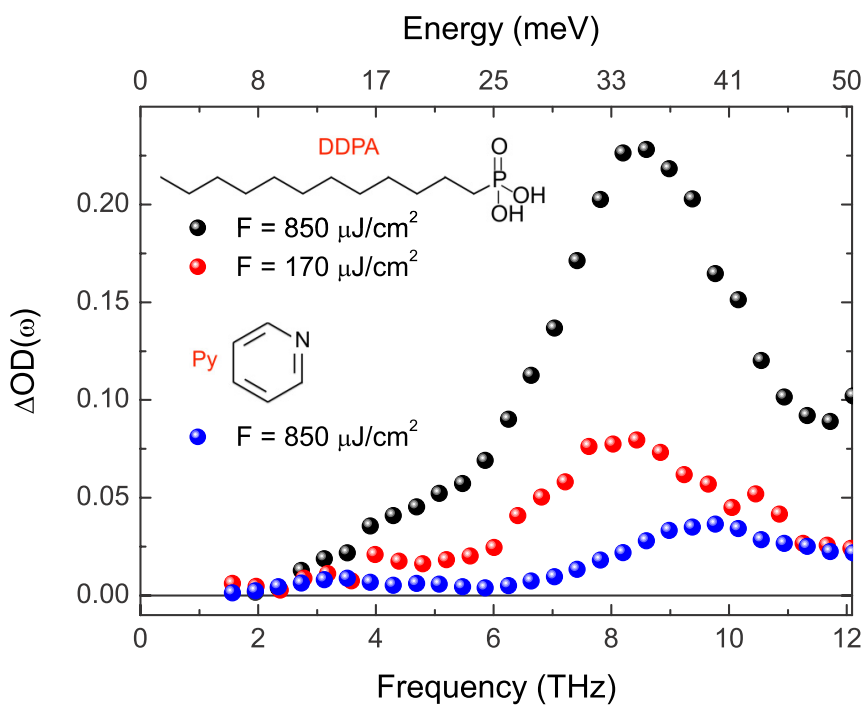

FIG. 3. (Color online) $\Delta \mathrm{OD}(\omega)$ spectra for the DDPA passivated rods, as well as the effect of partial ligand exchange of DDPA with pyridine. Pump fluences are indicated in the figure. 


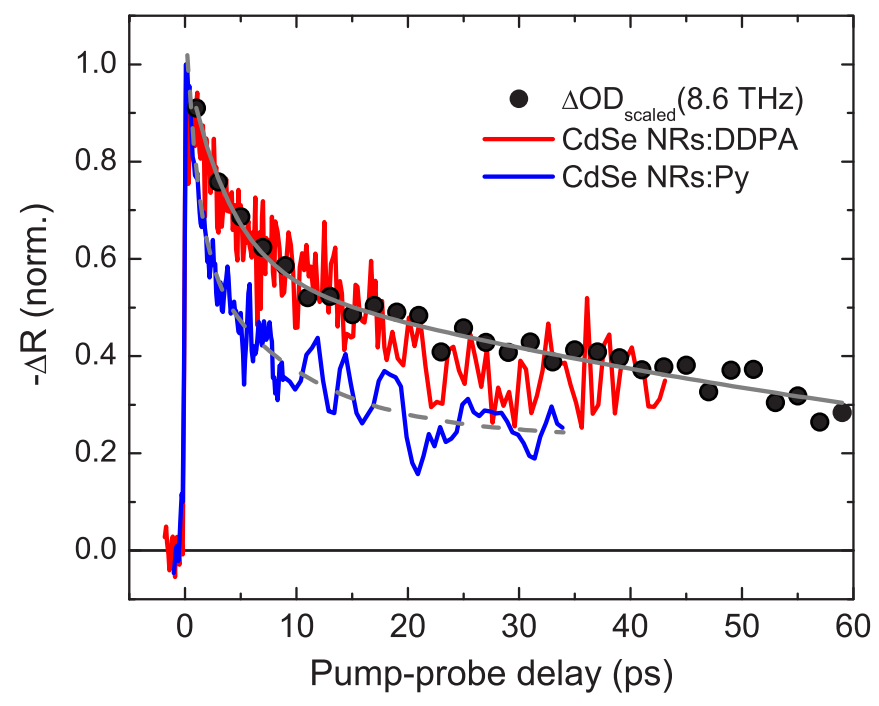

FIG. 4. (Color online) Normalized and scaled $\triangle \mathrm{OD}(8.5 \mathrm{THz})$ and the differential THz reflectance $[\Delta R(t)]$ dynamics for the DDPA and pyridine passivated $\mathrm{CdSe}$ nanorods.

of 5 reduction in pump fluence. No shift in the peak frequency is observed with reduced excitation density, inconsistent with a plasmon-Fröhlich phonon mode, ${ }^{25}$ and consistent with our assumption of there being no more than one exciton per nanorod in our model.

To follow the exciton relaxation dynamics, a onedimensional slice at the peak $(8.6 \mathrm{THz})$ of the $\Delta \mathrm{OD}$ spectra is shown in Fig. 4, demonstrating a clear biexponential decay with $42 \%$ of the signal undergoing a fast $4.0(7)$ ps decay and the remaining 58\% relaxing slower with a 90(8) ps decay constant. These relaxation times are comparable with previous timeresolved optical studies of CdSe nanorods. ${ }^{26,27}$ The change in exciton relaxation dynamics due to ligand exchange was also investigated by performing a less time-consuming differential reflectivity measurement, monitoring the peak of the differential THz waveform shown in Fig. 1 for the DDPA and pyridine ligand passivated nanorods. The normalized transients for both samples are shown in Fig. 4, which tracks the $\Delta R(\Delta t)$ transient for the CdSe:DDPA nanorod and confirms that $\Delta R(\Delta t)$ is proportional to the ground state population. Ligand exchange with pyridine noticeably decreases the lifetime of the exciton ground state, well described again by a biexponential decay with a 1.8(2) ps fast time constant and a slower 42(3) ps time constant not well resolved by our limited temporal range. The fast decay is therefore identified with rapid hole charge transfer and localization from the core to the pyridine molecule, forming a charge separated complex and leaving a core electron behind. The slower relaxation process could be influenced by exciton resonant energy transfer to defective rods that act as traps for exciton annihilation, ${ }^{28}$ which for quantum dots typically occurs on a $100 \mathrm{ps}$ to nanosecond time scale. ${ }^{29}$ Transfer rates could, however, be enhanced for nanorods by the aspect ratio-driven alignment of the rods when drop cast onto a film and we identify this as a topic of future investigations.

In conclusion, we have determined the picosecond dynamics of excitons in $\mathrm{CdSe}$ nanorods by probing their internal excitonic transitions at meV energies. The exciton ground state decays on two time scales, one dominated by surface trapping on a sub-5 ps time scale and another on a several tens of ps time scale. The dynamics of these processes are significantly influenced by the capping by organic ligands, with the transition strength suppressed and relaxation enhanced by introduction of the hole scavenging pyridine ligand. Several promising lines of investigation are now possible using these internal exciton transitions. Signatures of electron-phonon coupling are indicated when there are absorbance features occurring at the nanorod LO phonon frequency. The influence of surface ligands on dynamics as well as the energetics of the excitonic transitions will lead to a sensitive test of theory. Finally, as the temporal resolution is improved it will be possible to investigate directly the cooling of hot excitons through the densely packed manifold of states.

M.M.D. and D.G.C. acknowledge funding from NSERC. D.G.C. also acknowledges funding from CFI and FRQNT. The authors thank M. Kraus and P. Kambhampati for photoluminescence measurements of the nanorods. *cooke@physics.mcgill.ca

${ }^{1}$ D. V. Talapin, J.-S. Lee, M. V. Kovalenko, and E. V. Shevchenko, Chem. Rev. 110, 389 (2010).

${ }^{2}$ A. L. Efros and M. Rosen, Annu. Rev. Mater. Sci. 30, 475 (2000).

${ }^{3}$ P. Kambhampati, Acc. Chem. Res. 44, 1 (2011).

${ }^{4}$ R. A. Kaindl, M. A. Carnahan, D. Hägele, R. Lövenich, and D. S. Chemla, Nature (London) 423, 734 (2003).

${ }^{5}$ T. Suzuki and R. Shimano, Phys. Rev. Lett. 103, 057401 (2009).

${ }^{6}$ T. Suzuki and R. Shimano, Phys. Rev. Lett. 109, 046402 (2012).

${ }^{7}$ M. C. Beard, G. M. Turner, and C. A. Schmuttenmaer, Nano Lett. 2, 983 (2002).

${ }^{8}$ F. Wang, J. Shan, M. A. Islam, I. P. Herman, M. Bonn, and T. F. Heinz, Nat. Mater. 5, 861 (2006).

${ }^{9}$ M. C. Beard, G. M. Turner, J. E. Murphy, O. I. Micic, M. C. Hanna, A. J. Nozik, and C. A. Schmuttenmaer, Nano Lett. 3, 1695 (2003).
${ }^{10}$ E. Hendry, M. Koeberg, F. Wang, H. Zhang, C. de Mello Donegá, D. Vanmaekelbergh, and M. Bonn, Phys. Rev. Lett. 96, 057408 (2006).

${ }^{11}$ P. Guyot-Sionnest and M. A. Hines, Appl. Phys. Lett. 72, 686 (1998).

${ }^{12}$ C. Y. Wong, J. Kim, P. Sreekumari Nair, M. C. Nagy, and G. D. Scholes, J. Phys. Chem. C 113, 795 (2009).

${ }^{13}$ D. M. Sagar, R. R. Cooney, S. L. Sewall, E. A. Dias, M. M. Barsan, I. S. Butler, and P. Kambhampati, Phys. Rev. B 77, 235321 (2008).

${ }^{14}$ B. Zaks, R. B. Liu, and M. S. Sherwin, Nature (London) 483, 580 (2012).

${ }^{15}$ J. Y. Lek, L. Xi, B. E. Kardynal, L. H. Wong, and Y. M. Lam, ACS Appl. Mater. Interfaces 3, 287 (2011).

${ }^{16}$ D. G. Cooke, F. C. Krebs, and P. U. Jepsen, Phys. Rev. Lett. 108, 056603 (2012). 
${ }^{17}$ M. Kress, T. Löffler, S. Eden, M. Thomson, and H. G. Roskos, Opt. Lett. 29, 1120 (2004).

${ }^{18}$ J. Dai, X. Xie, and X.-C. Zhang, Phys. Rev. Lett. 97, 103903 (2006).

${ }^{19}$ K. Iwaszczuk, D. G. Cooke, M. Fujiwara, H. Hashimoto, and P. U. Jepsen, Opt. Express 17, 21969 (2009).

${ }^{20}$ G. Gallot, S. P. Jamison, R. W. McGowan, and D. Grischkowsky, J. Opt. Soc. Am. B 17, 851 (2000).

${ }^{21}$ D. M. Mittleman, R. W. Schoenlein, J. J. Shiang, V. L. Colvin, A. P. Alivisatos, and C. V. Shank, Phys. Rev. B 49, 14435 (1994). ${ }^{22}$ F. W. Wise, Acc. Chem. Res. 33, 773 (2000).

${ }^{23}$ H. Lange, M. Artemyev, U. Woggon, T. Niermann, and C. Thomsen, Phys. Rev. B 77, 193303 (2008).
${ }^{24}$ A. I. Ekimov, F. Hache, M. C. Schanne-Klein, D. Ricard, C. Flytzanis, I. A. Kudryavtsev, T. V. Yazeva, A. V. Rodina, and Al. L. Efros, J. Opt. Soc. Am. B 10, 100 (1993).

${ }^{25}$ B.-R. Hyun, A. C. Bartnik, W.-k. Koh, N. I. Agladze, J. P. Wrubel, A. J. Sievers, C. B. Murray, and F. W. Wise, Nano Lett. 11, 2786 (2011).

${ }^{26}$ M. B. Mohamed, C. Burda, and M. A. El-Sayed, Nano Lett. 1, 589 (2001).

${ }^{27}$ I. Robel, B. A. Bunker, P. V. Kamat, and M. Kuno, Nano Lett. 6, 1344 (2006).

${ }^{28}$ J. Lee, A. O. Govorov, and N. A. Kotov, Nano. Lett. 5, 2063 (2005).

${ }^{29}$ G. D. Scholes and D. L. Andrews, Phys. Rev. B 72, 125331 (2005). 